\title{
TITLE: MULTIMODE HYDRODYNAMIC STABIITY CALCULATIONS FOR NATIONAL IGNITION FACILITY CAPSULES
}

AUTHOR(S): Nelson M. Hoffman. Douglas C. Wilson, William S. Varnum, William J. Krauser. and Bernhard $\mathrm{H}$. Wilde

SUBMITTED TO: 12th International Conference on Laser Interaction and Related Plasma Phenomena. Senri Life Science Center, Osaka, Japan, 24 - 28 April 1995

\section{DISCLAIMER}

This report was prepared as an account of work sponsored by an agency of the United States Government. Neither the United States Government nor any agency thereof, nor any of their employees, makes any warranty, express or implied, or assumes any legal liability or responsibility for the accuracy, completeness, or usefulness of any information, apparatus, product, or process disclosed, or represents that its use would not infringe privately owned rights. Reference herein to any specific commercial product, process, or service by trade name, trademark, manufacturer, or otherwise does not necessarily constitute or imply its endorsement, recommendation, or favoring by the United States Government or any agency thereof. The views and opinions of authors expressed herein do not necessarily state or reflect those of the United States Government or any agency thereof.

By acceptance of this article, the publisher recognizes that the U.S. Government retalins a nonexclusive, royalty-free license to publish or reproduce the published form of thls contribution, or to allow others to do so, for U.S. Govemment purposes.

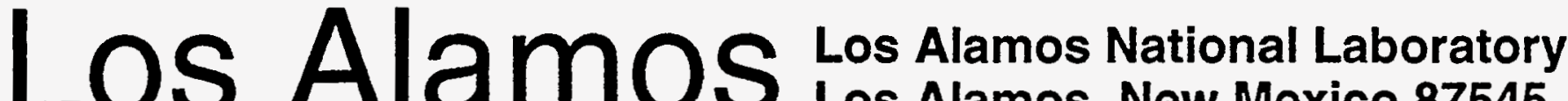 Los Alamos, New Mexico 87545}




\section{DISCLAIMER}

Portions of this document may be illegible in electronic image products. Images are produced from the best available original document. 


\title{
Multimode Hydrodynamic Stability Calculations for National Ignition Facility Capsules
}

\author{
Nelson M. Hoffman, Douglas C. Wilson, William S. Varnum, \\ William J. Krauser, and Bernhard H. Wilde
}

\author{
Thermonuclear Applications Group \\ Applied Theoretical and Computational Physics Division \\ Los Alamos National Laboratory, Los Alamos, New Mexico 87545 USA
}

\begin{abstract}
We examine the hydrodynamic stability of imploding ICF capsules by explicitly calculating the evolution of a realistic surface perturbation far into its nonlinear regime, using a 2D Lagrangian radiation-hydrodynamics code. The perturbation, which consists initially of mesh displacements in the capsule, is represented by the sum of many spherical harmonic modes, having finite amplitudes and realistic spectrum. A 90-degree sector of the capsule is modeled, allowing proper boundary conditions for all modes simultaneously. Because of the large distortion of the mesh that occurs during the calculations, it is necessary to rezone the mesh frequently, by mapping physical variables to a new undistorted mesh. No model-specific parameters are required in this technique. We have used the technique to calculate the yield of several designs for a National Ignition Facility capsule as a function of initial root-mean-square surface roughness $\sigma$ of the outer ablator surface or the inner cryogenic DT surface. Typically for a capsule we find a "cliff" at a critical value of $\sigma=\sigma_{\text {crit }}$ such that the yield of the capsule decreases abruptly for $\sigma>$ $\sigma_{\text {cril }}$, indicating a failure to ignite. The values of $\sigma_{\text {crit }}$ we compute are probably upper limits because of the lack of 3D effects, and inaccuracies in Lagrangian modeling of such unstable flows. It is expected that more accurate modeling. perhaps with 3D Eulerian codes, will lead to smaller values for $\sigma_{\text {crit }}$. We are beginning to carry out studies of the coupling of low-mode radiation flux asymmetries to higher-mode surface perturbations. We report also on sensitivity studies that examine the response of a capsule to small variations in the driving laser's power history. We find that realistic surfaces decrease a capsule's ability to tolerate drive variations.
\end{abstract}

\section{INTRODUCTION}

Hydrodynamic instabilities are processes that amplify small perturbations in the implosion of an ICF capsule, generally leading to a degradation in the performance of the capsule. If perturbations grow large enough, they may prevent fusion ignition by interfering with the compressional heating of the fusion fuel, for example, or by increasing the surface area through which heat is lost from the fuel, or by reducing the effectiveness of $\alpha$-particle trapping. With even more growth, the perturbations may generate hydrodynamic turbulence, which can transport mass, momentum, and energy in a way that disrupts the desired flow. 
To have confidence that our predictions of ignition are correct for National Ignition Facility (NIF) capsules, then, it is necessary that we have a sound theoretical and computational understanding of the effect of hydrodynamic instabilities in ICF implosions.

Several approaches have been used over the years to compute the effect of such instabilities. An early technique was to solve the linearized perturbed hydrodynamic equations, including energy flow by radiation and conduction, in a postprocessing mode (1). Later it was realized that the first-order flow could be calculated by a 2D nonlinear radiation-hydrodynamics code simultaneously with the zeroth-order calculation (2-4), obviating the need for a separate linearized perturbation code. We call these first-order single-mode (FOSM) calculations. One may compute the instability amplification factor of single spherical harmonic modes at some unstable interface by either of these approaches, assuming that the modes do not interact. Then one superimposes the modes linearly and determines the RMS deviation of the surface they define, giving a length scale $s$ over which materials are assumed to be mixed across the interface. The process, as outlined in Refs. 4 and 5, accounts for the weak nonlinearity by which neighboring modes influence one another's saturation, using a saturation parameter which must be normalized to experiment.

The mixing length scale $s$ obtained by this approach of linear mode superposition (LMS) is then used as input to a separate 1D calculation in which the capsule performance is finally determined. In recent implementations of this model, a heat-conduction multiplier is used in the 1D calculation to account for the fact that the thermal conductive flux across the unstable interface is enhanced as its area increases due to the growth of the perturbation.

\section{NONLINEAR MULTIMODE CALCULATIONS}

Recent evidence from theory and experiment $(6,7)$ points to a picture of latestage instability in ICF implosions which is at variance with the LMS picture. In the latter, materials are assumed to be thoroughly mixed at an atomic scale across unstable interfaces (5), throughout a region whose thickness is given by the mixing length scale $s$. The new evidence. however, suggests that unstable interfaces in ICF implosions are deformed into a network of polygonal bubbles surrounded by spike sheaths during the acceleration phase of the implosion. The bubbles occur with a range of sizes, but there is a dominant scale characterizing the largest and therefore most rapidly growing bubbles. The implication is that instability disrupts these flows, not by fine-scale mixing as in the LMS picture, but by large-scale effects such as those mentioned above, provided that the initial perturbation is not too large.

To model the bubble-network picture of hydrodynamic instability. we have performed calculations in which the unstable flow is represented directly with as much detail and as few modeling approximations as possible, given the constraints of finite machine memory and two-dimensionality. We use the same type of 2D radiation-hydrodynamics code that is used for the FOSM calculations described above, but our approach differs in several ways: many spherical harmonic modes (up to 24 thus far) are present in a single calculation; the amplitudes of the modes are realistic, not infinitesimal; and a 90-degree quadrant of the capsule is represented, to allow proper boundary conditions for all modes simultaneously. 
Furthermore, unlike the LMS approach, we do not run a separate calculation to model capsule performance, since the yield and other attributes of the capsule are calculated at the same time as the perturbation growth. Thus we avoid assumptions about mode saturation or enhanced heat flow, since we calculate these effects explicitly, and have no need for model parameters that adjust their magnitudes.

Two-dimensionality introduces at least two shortcomings in the calculations, one of which we can compensate for, while the other we cannot. First, a 2D surface is inherently less rough than a 3D surface with the same spherical harmonic spectrum, because of its axial symmetry. This is reflected in the expressions for the RMS deviation of such surfaces. For an isotropically rough 3D surface,

$$
\sigma_{3 D}^{2}=\frac{1}{4 \pi} \sum_{l}(2 l+1) R_{l 0}^{2}
$$

while

$$
\sigma_{2 D}^{2}=\frac{1}{4 \pi} \sum_{l} R_{l 0}^{2}
$$

for a 2D surface, where $R_{l 0}$ is the amplitude of the spherical harmonic $Y_{l}^{0}$. The factor $2 l+1$ in the expression for $\sigma_{3 D}$ arises from the azimuthal roughness in a 3D surface, and clearly has the effect of increasing the importance of higher mode numbers in 3D relative to 2D. To try to compensate for this effect in our calculations, we modify the relative amplitude of all modes in the $2 \mathrm{D}$ spectrum by the factor $2 l+1$, while keeping the total $\sigma$ constant. This modified spectrum we call a "bandwidth-equivalent-RMS" (BER) spectrum, because it has not only the same total $\sigma$ as the $3 \mathrm{D}$ spectrum which it is supposed to represent, but each mode. or band of modes, makes the same relative contribution to the total $\sigma$ as in the $3 \mathrm{D}$ spectrum.

The other shortcoming arises because of the fact that 2D bubbles grow more slowly than 3D bubbles. This is called the "shape effect" (7), and was pointed out by Layzer ( 8 ). The terminal velocity of a bubble is about $50 \%$ higher in 3D than in $2 \mathrm{D}$, for large density ratios. There is no obvious way to compensate for the shape effect in a 2D calculation, so a resolution must await 3D calculations. In the meantime, we take the viewpoint that 2D calculations furnish a lower limit to the magnitude of the disruptive effects of a perturbation with a given initial $\sigma_{0}$, and therefore our conclusions about the maximum tolerable $\sigma=\sigma_{\text {crit }}$ are actually upper limits to its value.

Another issue raised by our nonlinear multimode calculations is that of Lagrangian mesh distortion. Because the mode amplitudes are finite, the perturbation at an interface can eventually grow to seriously disturb the capsule implosion. In this case the distinction between zeroth-order and first-order flow is lost, distinct bubbles and spikes form in the density structure, and the mesh becomes greatly deformed. The code's Courant time step drops to an extremely small values or the implicit radiation-diffusion algorithm fails, halting the calculation.

To continue the calculation requires rezoning, that is, defining a new, more regular mesh, and mapping the physical variables from the old deformed mesh to the new regular mesh. To define the new mesh, we use a remeshing technique 
due to Brackbill and Saltzman (9), which allows the mesh to adapt to steep gradients in physical variables, solving an elliptical equation to define the new mesh lines using the old mesh as a boundary condition. Then a 2nd-order-accurate technique due to Scannapieco (10) is used for the remapping of variables. By repeated application of the rezoning procedure we can follow flow patterns which are quite turbulent. It is clear, however, that the calculated performance of a capsule, in particular its yield, can be affected by the exact prescription for rezoning (e.g., the time at which rezoning begins, the frequency with which it is carried out, and the degree to which the new mesh is allowed to approach the exact Brackbill-Saltzman solution). The sensitivity is greatest near "cliffs" in initial surface roughness, i.e., when $\sigma_{0}$ is near $\sigma_{\text {crit }}$. The effect is to make the location of the cliff uncertain to some degree. We are currently assessing the relative accuracy of the various prescriptions for rezoning, through comparison to experiments and theory. In the meantime, we will simply conclude that rezoning is required to allow these calculations to continue through the time of fusion burn of the capsule, but introduces some uncertainty into the results.

\section{NIF CAPSULE CALCULATIONS}

We have applied the nonlinear multimode technique to several NIF designs, in order to specify the maximum tolerable $\sigma_{0}$ of various surfaces in the capsule. Figure 1 shows the initial perturbation of the inner deuterium-tritium (DT) ice interface in one calculation of the "PT" design (11), where the perturbation is the sum of 24 spherical harmonic modes $(l=2,4,6, \ldots, 48)$. The modal amplitudes are given by a BER spectrum obtained by scaling a ID Fourier analysis of cryogenic DT data to a $2 \mathrm{D}$ power spectrum, and then applying the BER enhancement discussed earlier.

Calculations using the BER spectrum are still in progress, but we have carried out other studies of the PT capsule with spectra that are simply scaled directly from the $2 \mathrm{D}$ power spectrum of DT ice surfaces without the BER enhancement, to determine the effect of spectral shape on our conclusions about the maximum tolerable surface roughness. In these studies, we have taken a particular initial spectrum and scaled it up or down to vary $\sigma$. As Fig. 2 shows, the yield of the PT capsule is relatively insensitive to $\sigma$ until it reaches a critical value, $\sigma_{\text {crit }}$, above which the yield falls dramatically, indicating a failure to ignite. Open circles in Fig. 2 show results for an 8-mode perturbation with relatively high modes only $(l=12,16,20, \ldots, 40)$, while the filled circles show results for a 24 -mode spectrum with low modes included $(l=2,4,6, \ldots, 48)$. The location of the cliff, $\sigma=\sigma_{\text {crit }}$, increases for the spectrum with low modes included, because low modes grow less rapidly than high modes and produce less disruption of the flow. The scatter in the calculated yields near the cliffs is caused by variations in the prescription for rezoning. The dashed lines indicate a lower limit to the calculated performance, which we adopt at present as the most cautious estimate.

Using the technique of direct nonlinear multimode instability simulations, we can now begin to examine the coupling of various asymmetries and departures from ideal driving conditions, simultaneously or taken a few at a time. For example, we can incorporate the low-mode radiation drive asymmetry, determined by an integrated hohlraum calculation, into our instability calculations, to examine its coupling to surface perturbations. Such studies are now in progress. We can perturb two or more surfaces simultaneously, to examine how such per- 


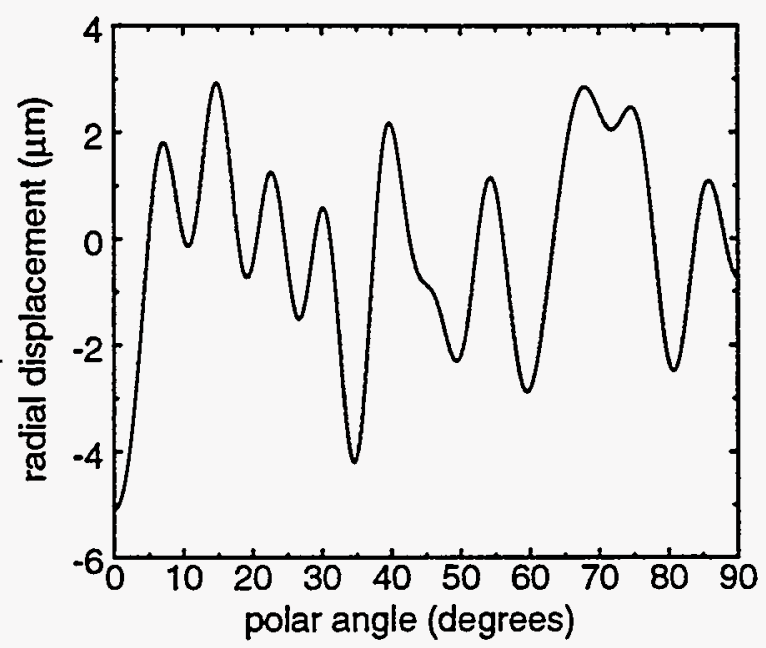

Figure 1. Initial 24-mode BER perturbation on inner DT ice interface, with $\sigma=1.75 \mu \mathrm{m}$.

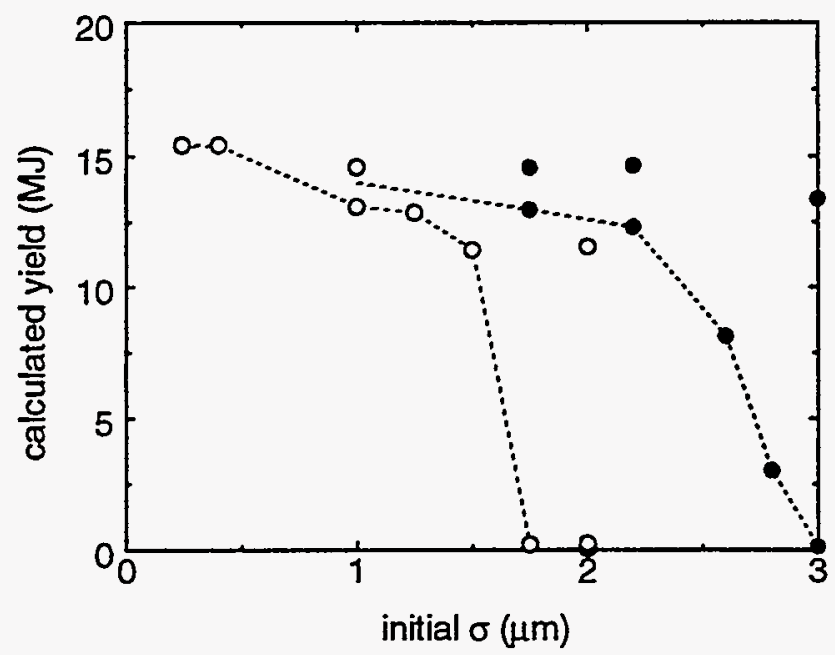

Figure 2. Variation of yield with DT ice inner surface roughness, for an 8-mode perturbation (open circles) and a 24-mode perturbation (filled circles).

turbations couple and reduce the allowable roughness at either surface. We can study the sensitivity of a capsule to variations in the hohlraum drive history, as is often done in 1D, but now include the non-ideal circumstances of surface roughness and drive asymmetry. Figure 3 shows results of such a study, in which the yield of a NIF capsule is determined as a function of the temperature in the final drive pulse. Open circles show the standard 1D result, which assumes perfect smoothness of all capsule surfaces. The filled circles show the effect of roughness with $20 \mathrm{~nm}$ RMS on the outer surface of the ablator, about the limit of our current fabrication capability. Most of the capsule's ability to tolerate low drive is negated by surface roughness. Future studies will include drive asymmetry and perturbations on all surfaces simultaneously, when examining sensitivity to drive variations. 


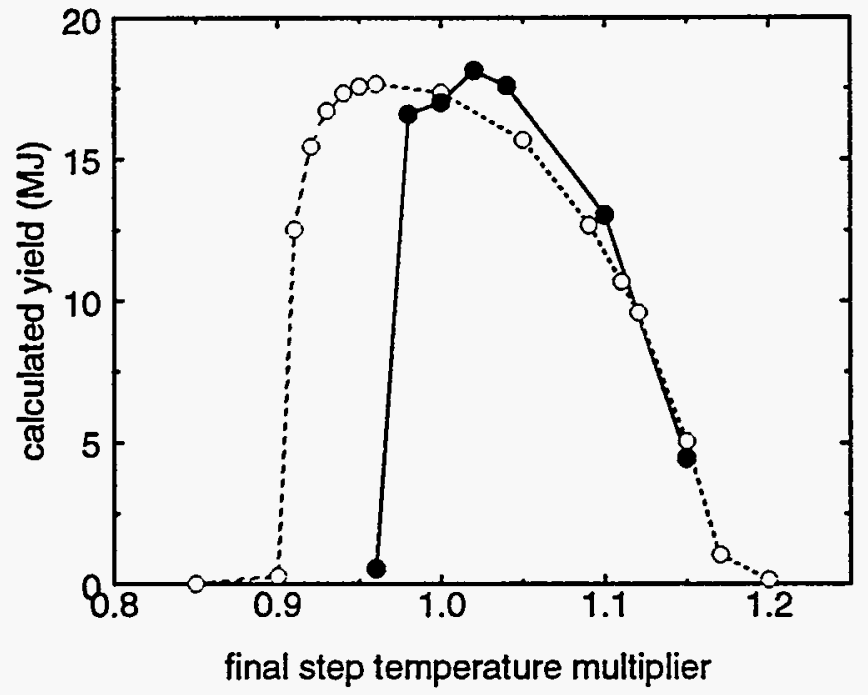

Figure 3. Drive sensitivity profiles for NIF capsule. Open circles show standard 1D result. Filled circles show result of $2 D$ calculations with ablator roughness of $20 \mathrm{~nm}$.

\section{ACKNOWLEDGMENTS}

We are grateful to Steve Haan for pointing out the necessity of using bandwidth-equivalent-RMS spectra in 2D calculations, and for suggesting the calculations which led to this work in the first place. We also thank Bill Powers for improvements and advice in the use of the Brackbill-Saltzman remesher. This work was supported under USDOE contract W-7405-ENG-36.

\section{REFERENCES}

1. McCrory, R. L., Morse, R. L., and Taggart, K. A., Nucl. Sci. Eng. 64, 163-176 (1977); Scannapieco, A. J., and Cranfill, C. W., "A Derivation of the Physical Equations Solved in the Inertial Confinement Stability Code DOC," Los Alamos Scientific Laboratory report LA-7214-MS (1978).

2. Verdon, C. P., McCrory, R. L., Morse, R. L., Baker, G. R., Meiron, D. I., and Orszag, S. A., Phy's. Fluids 25, 1653-1674 (1982).

3. Munro, D. H., Phys. Fluids B 1, 134-141 (1989).

4. Haan, S. W., Phys. Rev. A 39, 5812-5825 (1989).

5. Dittrich, T. R., Hammel, B. A., Keane, C. J., McEachern, R., Turner, R. E., Haan, S. W., and Suter, L. J., Phys. Rev. Lett. 73, 2324-2327 (1994).

6. Remington, B. A., Weber, S. V., Haan, S. W., Kilkenny, J. D., Glendinning, S. G., Wallace, R. J., Goldstein, W. H., Wilson, B. G., and Nash, J. K., Phys. Fluids. B 5, 2589-2595 (1993).

7. Dahlburg, J. P., Bull. APS 39, 1576 (1994); Shvarts, D., Bull. APS 39, 1576 (1994).

8. Layzer, D., Astrophys. J. 122, 1-12 (1955).

9. Brackbill, J. U., and Saltzman, J. S., J. Comp. Phys. 46, 342-368 (1982).

10. Scannapieco, A. J., "Automatic Rezoner for Lagrangian Quadrilateral Hydrocodes," Los Alamos National Laboratory report LA-UR-82-2897 (1982).

11. Haan, S. W., Bull. APS 39, 1635 (1994). 